\title{
Connection Mode Optimization of Power Distribution Network Based on Load Fusion and Hierarchical Control Theory
}

\author{
Bingqian Song \\ North China Electric Power University, Beijing, 102206 \\ alanncepu@foxmail.com
}

Keywords: Load fusion; Decision level fusion; IPDI; SRM; Comprehensive evaluation algorithm

\begin{abstract}
With the complex and variable structure and operation mode of power distribution network, the traditional load area model is difficult to adapt to the distribution network transformation. Therefore, based on the idea of information fusion, this paper puts forward a kind of load fusion theory, and constructs three level hierarchical structure (load level)of organization level, coordination level (decision level), to carry out Decision - level and load - level fusion for the main area of the load and load level fusion for new fusion area, using the comprehensive evaluation algorithm to optimize the connection mode of power distribution network, and design a complete wiring scheme, which has a good reference value for the power distribution network planning.
\end{abstract}

\section{Introduction}

With the development of distribution network and the progress of science and technology, the structure and operation mode of distribution network become more and more complicated and changeable, and the reliability and economy of distribution network are put forward. Due to the continuous development of control technology, the control method is becoming more and more diversified, especially the development of sensor technology and the electronic chip, in the electric power, metallurgy, chemical industry, building materials and other industry information fusion technology has been widely used, mainly used with detection or monitoring and diagnosis system, intelligent robot systems, intelligent manufacturing system, modernization of management, and multimedia system, meet the users of the system of high performance requirements.

According to the actual situation of power distribution network load, combined with the application of fusion theory in many fields, according to the model of classical intelligent control system, the concept of load combination and its model are proposed. Load combination is in the distribution network planning considering the distribution of the load in the area and the load level, the regional topography, municipal construction and other related factors, the load distribution in the region is fused to judge, and then the whole area of the load information fusion processing, and finally come to the need of the results. At present, some scholars in China and abroad have studied the integration of load and power system automation control in the field of power plant, distribution network, user side and so on. The theory of automatic control is applied to the promotion of automation and intelligent level of power system and the researches on hierarchical control theory and automation of power system which is carried out in document 1; Based on the hierarchical structure of self-healing control of urban power grid, the system framework of the self-healing control of urban power network is designed, and the structure and function of all levels of smart structures are designed; In document 2, based on the hierarchical structure of self-healing control of urban power grid, the system framework of the self-healing control of urban power network is designed, and the structure and function of all levels of smart structures are designed; Literature 3 raises the practical application of hierarchical theory on governor, then combining with the PCC controller hardware and software characteristics, the specific application of the theory is described in detail from the hardware structure design, functional configuration. 


\section{Basic Principle}

In 1977, according to the information fusion theory, G.N. Saridis proposed a kind of three level hierarchical structure of intelligent control for robot control. Whether it is information analysis, or behavior control, there are levels. Upper is mainly imitate human's behaviors, based on the knowledge of the system responsible for the macro planning, decision-making and learning, data storage, task coordination, lower layer is responsible for the implementation of specific control task, the numerical operations, the function of each module can be assigned to the subjective probability model and fuzzy set. Its basic control principle is IPDI (Increasing Precision with Decreasing Intelligence) principle, namely, the degree of precision increasing from top to bottom, intelligent degree by reduction. The hierarchical control system is composed of a three layer structure, the main content of the three layer structure including three levels: organization level, coordination level and execution level, and the cascade structure are shown in Fig. 1.

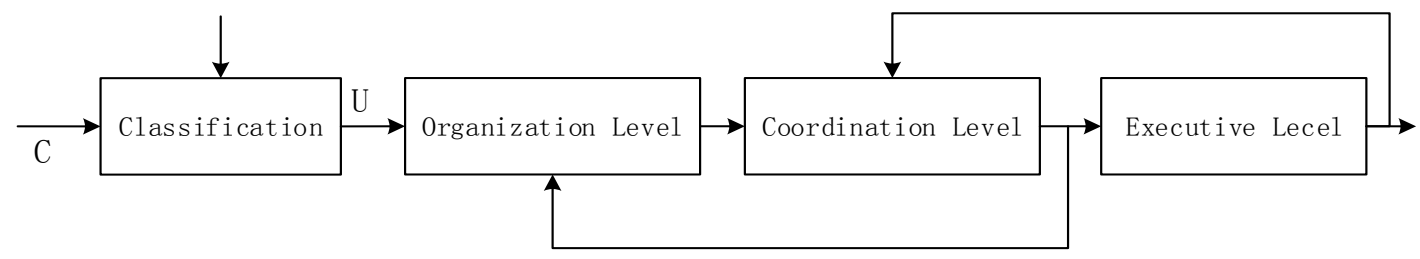

Figure 1. Hierarchical cascade structure

Corresponding load fusion model can be divided into three levels, followed by the organization level fusion, decision level fusion and load level fusion, the hierarchical control system schematic diagram in Fig. 2.

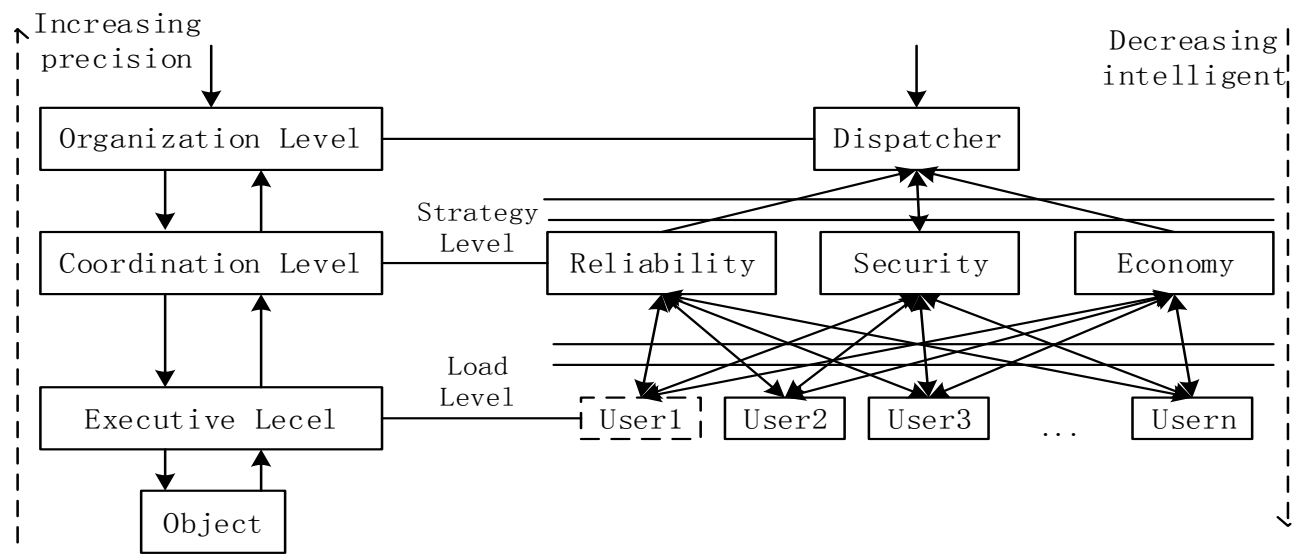

Figure 2. Three layer structure in hierarchical control system of Load fusion model

Organizational Level. The organization level, the top layer of the load fusion, its role is to accept a given external commands and tasks, the use of task distribution, to find the combination of the subsystem control tasks to complete the task; Then these sub tasks are required to be delivered to the decision-making level, through processing decision level, finally, the specific implementation requirements sent to the load level to complete the required tasks; Finally, the results of load level implementation are evaluated, and the evaluation results are gradually up to the top. This is a typical information fusion and artificial intelligence problem to get the solution, which have been many experts have made a lot of work, in literature 7 introduces Moed and Saridis proposed by Boltzmann machine neural network (BM) network method to realize the organization's function. The neural application network energy function as the cost function, through minimization, finds the optimal state. In the selection process of the distribution network connection, load level fusion of data is input nodes, eventually the decision results of evaluation as output nodes, used to achieve the load between the input and output nodes of the link between the hidden nodes. The 
implementation of the task is only the best way to match the connection, so the organization level structure is relatively simple.

Decision Level. Decision - level fusion is a kind of high - level fusion, which directly provides the basis for the distribution network planning. Therefore, the decision level fusion must be based on the actual demand of the decision problem, and makes full use of all kinds of load characteristic information provided by the load level fusion and other aspects index using appropriate fusion techniques to achieve. In decision level attribute fusion structure (Fig. 2), each evaluation index and feature information should form a feature vector, and then use a certain combination of evaluation algorithm to combine these feature vectors to form a joint feature vector, getting attribute decision based on combined feature vectors. Decision level fusion is the final result of load fusion, and the final planning scheme of the distribution network is developed.

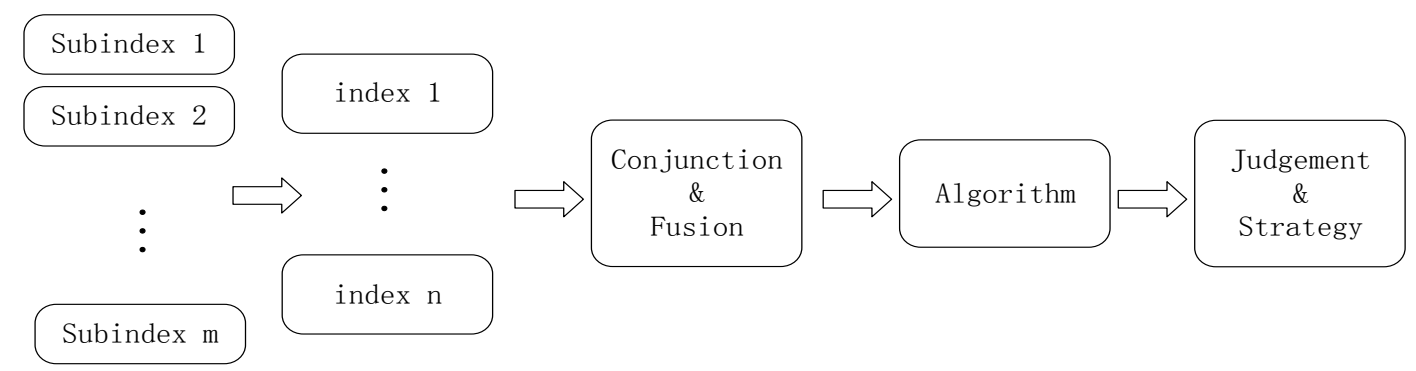

Figure 2. Attribute fusion structure of decision level

Load Level. Load level fusion is mainly considering the division and combination of the load in the region, and the data of the load itself and make load distribution diagram. According to the planning area distribution network voltage level is different, the electricity area divided into rules district (grid) or irregular cell (substation and feeder area), each cell load types of a single, unified planning, district in the area of integration has become a central; central integration has become a large area. In the area of central and large areas, a variety of factors to reconsider the load.

Load level fusion uses structural risk minimization (SRM) induction principle to find the best fusion method. As shown in Fig. 3, in an ideal model of the regional scope of a 110kV substation, it is divided into 24 areas, namely, the observation set is $\{z 1, z 2, \ldots, z 24\}$, for fusion selection. the goal is to find a combination of the best combination of economic, reliability and security aspects $\mathrm{Sn}$. Calculation method is: the beginning from $\mathrm{Z1}$, according to the order of the $110 \mathrm{kV}$ substation from the near to the distant, one by one to fuse calculation (see ref. 8), calculated the best fusion region set.

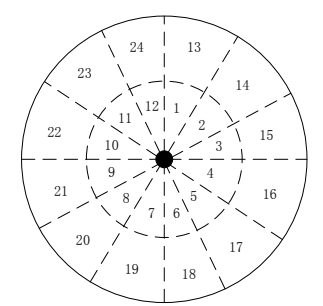

Figure 3. 110kV substation area load level fusion model

The load ratio calculation formula for load level fusion is

$$
\eta_{n}=\frac{\sum_{i=1}^{n} S_{i} \rho_{i}}{P} \quad n=1,2, \ldots, 24
$$

Where: Load rate of $110 \mathrm{kV}$ substation after adding the first $\mathrm{n}$ load;

$\mathrm{Si}$ is the area of the first I cell; Load density for the first I cell; Capacity P is $110 \mathrm{kV}$ substation. 


\section{Optimization Model of Power Distribution Network Connection Mode}

This section will combine the actual situation of distribution network according to the idea and model of load fusion, offering planning optimization scheme of distribution network connection mode. According to the load fusion model, the scheme is divided into the main area and the fusion region.

Region Division. Determine the shape of the connection area on the basis of the load level fusion. $110 \mathrm{kV}$ substation will be divided into different areas, according to the districts load level; the district will be combined into different areas. Then according to the location, shape, load density, the central area is integrated into an $110 \mathrm{kV}$ substation as the whole region. The region in the ideal state can be seen as a circular or regular hexagon. According to the actual characteristics of the urban distribution network, take an analytical model which can be adjusted of the power supply area, as shown in Fig. 4. According to the diagonal line, the model area is divided by the dotted line. The center of the solid round indicates $110 \mathrm{kV}$ substation location.

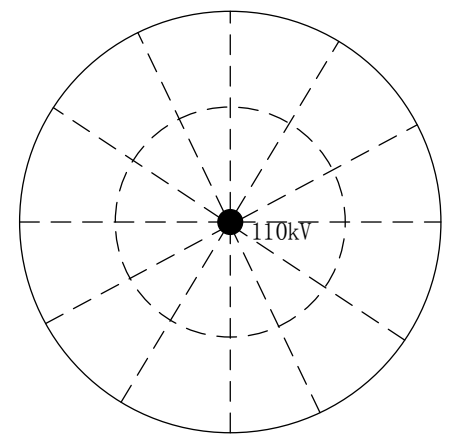

Figure 4. Main body area model of $110 \mathrm{kV}$ substation

Considering some of the new fusion area, under the circumstances that this part of the district which is in the main $110 \mathrm{kV}$ substation area has been formed, Plan and adjust of the connection mode of the new adding cell load. Assuming that the main region has adopted a three section three contact wiring connect ways, and the new area of the integration is circle, the center for the $10 \mathrm{kV}$ substation location, as shown in Fig. 5.

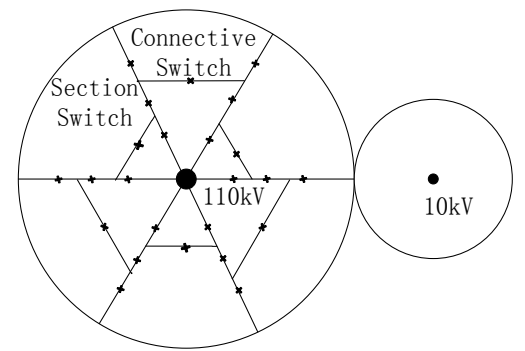

Figure 5. New fusion cell model of $10 \mathrm{kV}$ substation

Best Selection of Connection Mode. In accordance with the above methods to divide the region, subject area and the new regional integration in two stages to choose the mode of connection, both use comprehensive evaluation system, flexibility, reliability and economy as the main boundary conditions and judgment and choice of connection mode, ultimately determine the entire $110 \mathrm{kV}$ substation power supply area in the best way of wiring.

When the main area are in the selection of wiring, the organizational level integration is just an information transfer channel, so do not consider the integration of organizational level, according to the second section of the narrative method of load level fusion and decision level fusion. The new fusion region, which is mainly used in the distribution network planning and transformation, has been carried out after the main area has been planned. Due to the factors that have been taken into account in the substation load rate, the scheme design is not used for load level fusion. Because of the small power supply in the fusion area, the economic cost is low when the line is set up, therefore, 
in the comprehensive evaluation; the indicators of reliability and flexibility are main, supplemented by economic indicators.

In this paper, we design several new connection modes, and then calculate the economic index $\left(F_{\mathrm{N}}\right)$, reliability index (SAIFI, SAIDI, ASAI) and flexibility index (Capacity Reserve), and the TOPSIS method is used to make a comprehensive evaluation of each connection mode, then obtains the planning of the regional integration scheme. As shown in Figure 6, when the main area for the three section three contact wiring, the best way of wiring in the fusion area are the radial continuation power supply scheme and the local loop network power supply scheme.

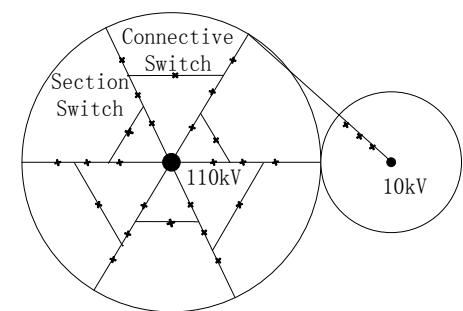

(a)Radial continuation power supply scheme

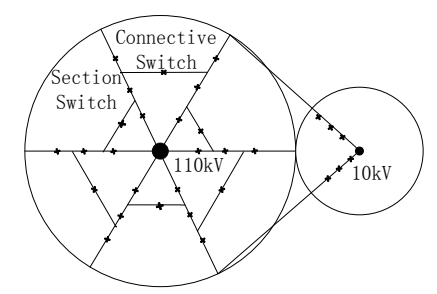

(b) Part ring network power supply scheme

Figure 6. Power supply scheme in the new fusion area

\section{Conclusion}

In the medium voltage distribution network planning, the traditional load area model is a standard round or regular hexagon, which is actually in the different stages of the distribution network construction. Due to the development of economy, the shape of the load area and the type of load change, the load area are not completely consistent with the traditional model. Therefore, the traditional model cannot meet the needs of the actual work in the distribution network planning and transformation process. The dynamic change of load area is considered in the load fusion theory. The connection mode is divided into execution level three levels of organizational level and decision level, and gives the partition domain phase of the planning method, according to their respective load level fusion and decision level fusion to select the best connection mode of $10 \mathrm{kV}$ power distribution network. Through the analysis and calculation of the design model, a complete central wiring scheme is obtained, which has a good reference value and reference value for the future distribution network planning.

\section{References}

[1] Feng Jinbing, Hierarchical control theory and power system automation research, Electronics World, 2012(22).

[2] Yu Kun, Study on self-healing control of the urban power grid based on hierarchical control theory, 2011.

[3] CAI Wei-jiang, Yu Ji-wei, Chen Xiao-yong, Cai Xiao-feng, Application research of hierarchical control in water turbine governing system, Mechanical \& Electrical Technique of Hydropower Station,2012(06).

[4] Saridis G N.Intelligent robot control. IEEE Transactions on Automatic Control. 1983.

[5] Saridis G N.Analytic formulation of the principle of increasing precision with decreasing intelligence for intelligent machines. Automatica. 1989.

[6] Saridis G N.Entropy Formulation of Optimal and Adaptive Control. IEEE Transactions on Automatic Control. 1988.

[7] Li Shaoyuan, Wang Jingcheng. Intelligent control (Second Edition). Mechanical Industry Press, 2005. 
[8] N. Vapnik Vladimir, Zhang Xuegong. The essence of statistical learning theory. Tsinghua University press, 2000.

[9] Tsao, Teng-Fa,Chang, Hong-Chan. Composite reliability evaluation model for different types of distribution systems. IEEE Transactions on Power Systems. 2003.

[10]Y.G. Sun, W.L. Li, D.S. Dong, X. Mei, H.Y. Qiang, "Dynamics Analysis and Active Control of a Floating Crane", Tehnicki Vjesnik-Technical Gazette, vol.22, no. 6, pp. 1383-1391, 2015.

[11][Seng TL, Khalid MB, Yusof R. Tuning of a Neuro-Fuzzy Controller by Genetic Algorithm. IEEE Trans. on Systems, Man, and Cybernetics, 1999, 29(2): 226 236. 